\title{
Research and Analysis of the Effect of Refined Nursing Intervention on COVID-19 Epidemic in Hemodialysis Center Runing Title:Effect of Refined Nursing Intervention on COVID-19 Epidemic
}

\author{
Qinglai Zhang ( $\nabla$ zhangqinglaicf@163.com ) \\ Chaoyang Hospital Affiliated to Capital Medical University \\ Shuo Wang \\ Chaoyang Hospital Affiliated to Capital Medical University \\ Yue Zhang \\ Chaoyang Hospital Affiliated to Capital Medical University \\ Fei Meng \\ Chaoyang Hospital Affiliated to Capital Medical University
}

Research article

Keywords: COVID-19, Maintenance hemodialysis, Fine nursing intervention, Intervention strategy, SCL - 90.

Posted Date: November 17th, 2020

DOI: https://doi.org/10.21203/rs.3.rs-108402/v1

License: (c) (i) This work is licensed under a Creative Commons Attribution 4.0 International License. Read Full License

Version of Record: A version of this preprint was published at BMC Nursing on April 26th, 2021. See the published version at https://doi.org/10.1186/s12912-021-00584-5. 


\section{Abstract}

Background To analyze and discuss the effect of clinical refined nursing intervention on maintenance hemodialysis patients during the outbreak of COVID-19.

Methods The Symptom Checklist90 (SCL-90) was used to conduct nursing interventions before, during and after dialysis for maintenance hemodialysis patients, and the results were compared with the Chinese adult SCL-90 norm.

Results The scores of all factors of SCL-90 in maintenance hemodialysis patients are all higher than normal Chinese SCL-90, and patients with a single factor score greater than or equal to 2 have a higher proportion of depression and anxiety, with extremely significant difference $(\mathrm{P}<0.01)$. The depression and anxiety of the patients were reduced after the intervention, and there was a statistical difference. Two nucleic acid test results of 172 patients were negative.

Conclusion During the COVID-19 epidemic, providing maintenance hemodialysis patients with refined nursing intervention can improve their health behavior compliance, regulate negative emotions, reduce related complications, improve their quality of life, and improve the nurse-patient relationship. At the same time, it can fundamentally control the occurrence of nursing errors and adverse events, with outstanding clinical application value.

\section{Background}

In early 2020, the sudden outbreak of the novel coronavirus pneumonia broke out in Wuhan and quickly spread across the country. Coronavirus Disease 2019 is abbreviated as COVID-19. It is a new infectious disease caused by a new type of coronavirus that has the epidemiological characteristics of strong contagion, multiple routes of transmission, and wide spread ${ }^{[1-3]}$. COVID-19 is an infectious disease spread through respiratory droplets or contact such as coughing and sneezing. The disease is transmitted from person to person, with rapid onset, strong infectivity, rapid changes in the course of disease, and people are generally susceptible to it. Because maintenance hemodialysis populations have low self-immunity, poor resistance and most of them suffer from basic diseases, they are easily susceptible to the novel coronavirus ${ }^{[4]}$. Once infected, the symptoms are severe and difficult to treat. The outbreak of novel coronavirus pneumonia has brought enormous psychological pressure to the patients undergoing hemodialysis, resulting in unhealthy psychological emotions ${ }^{[5]}$ such as anxiety and depression. Therefore, it is of great significance to carry out strict management and fine nursing intervention for maintenance hemodialysis patients during the prevention and control of novel coronavirus. The report is as follows.

\section{Methods}

\subsection{Research objects}

Using a random questionnaire survey method, during the novel coronavirus epidemic, 172 people from the center's maintenance hemodialysis patients were included to participate in this survey. Inclusion criteria: dialysis treatment started more than 2 months ago; hemodialysis was conducted three times a week; there was no language communication and cognitive impairment, and they could express their wishes accurately; the patients had informed consent. Exclusion criteria: patients with Alzheimer's disease or other mental disabilities; patients with malignant tumors; patients with a history of mental illness; patients with limb paralysis; patients who refused to participate in this study. The investigation time is from 2020.2.1 to 2020.7.1.

\subsection{Methods}

\subsubsection{Symptom Checklist90 (SCL-90)}

SCL-90 scale is the most widely used psychological disease examination scale at present. It involves from feeling, emotion, thinking, consciousness, behavior to living habits, interpersonal relationship, diet and sleep, including 9 subscales with 90 items, which were divided into 10 categories, namely 10 factors: somatization (reflecting subjective physical maladaptation), obsessive-compulsive symptoms, interpersonal sensitivity, depression, anxiety, hostility, paranoia, psychosis, and additional factors (reflecting sleep and appetite). Scoring criteria: a five-grade scoring system (from 1 to 5) was adopted, from none to serious. Add the scores of each item of 90 items to get the total score. Total average score $=$ total score $/ 90$. Factor score $=$ the total score of each item constituting a factor $/$ the number of items constituting a factor. The score range is $1-5$, the lowest is 1 , and the highest is 5 . The higher the score, the more obvious the symptoms ${ }^{[6]}$ and the worse the mental health.

\subsubsection{Refined Nursing Intervention Methods}

\subsubsection{Nursing intervention before dialysis}

(1) Improve the nursing concept To implement a refined nursing model for maintenance hemodialysis patients, it is necessary to start with improving the nursing concept of the nursing staff. The nursing staff adhere to the principle of precision and refinement in the process of 
nursing intervention, be careful in their daily work and miss no details. They need to have the right work attitude and provide patients with highquality nursing services. The center cancels collective shifts and holds group work meetings twice a day. The head nurse personally presides over the meeting. Each group leader participates in the meeting. The head nurse conveys the department's prevention and control requirements during the novel coronavirus epidemic and analyzes the spirit of related policies. The current difficulties faced by the team, urgent problems to be resolved, changes in critically ill patients' condition, difficult vascular access, and other issues were put forward by group leader at the meeting to jointly study solutions, and according to the dynamic changes of the epidemic situation, the problems in the management are analyzed and continuously improved. Among the problems reported by each group, more than $90 \%$ are the psychological pressure caused by the epidemic on dialysis patients, causing the patients to have negative psychological emotions. In response to this problem, the head nurse leads the nurses to conduct psychological counseling for dialysis patients, correct their negative psychological emotions and resolve contradictions. Do what should be done today, handle the problem in the bud, effectively relieve the psychological pressure of patients, and ensure the normal progress of hemodialysis treatment.

(2) Strengthen the training of nursing staff If the professional level of nursing staff is not up to standard, it will hinder the implementation of fine nursing model. Therefore, the department regularly strengthens the training of nursing staff. The training content is mainly based on theoretical knowledge and clinical nursing practice. While enriching the nursing staff's theoretical knowledge, improve the nursing staff's clinical practice ability, and improve the nursing staff's professional quality as a whole. During the epidemic, all medical staff of blood purification participated in the COVID-19 related trainings in Beijing and the hospital as required and met the standards.

(3) Strengthen prevention and control measures The dialysis room strengthens prevention and control measures, and formulate prevention and control plans: isolation of the blood purification center area, closed management of the dialysis room, strict regulation on two closures, that is, closure of the front door of dialysis room and closure of the treatment area. Family members are not allowed to enter the room and for patients who cannot take care of themselves, all the care work is taken by the responsible nurses and caregivers. Dialysis patients get on and off the machine at staggered peak, and issue special dialysis certificate, vehicle license, fixed family member, fixed vehicle, fixed shift, fixed area, strict division, allocated machines and time interval to avoid personnel gathering. Ensure site cleaning before dialysis, strict closed-loop terminal disinfection, strict and correct hand washing, correct wearing of masks, and two-point one-line travel route. Set up pre-inspection team, the inspection content is dynamically linked to the epidemic situation, the inspection scope includes dialysis patients and their families, the order of investigation is: test temperature, health code display, nucleic acid test report within 7 days, and ask the patients about their travel trajectory during non-dialysis days. The body temperature of dialysis patients should be tested 4 times on the day of dialysis (before admission, before dialysis, 1 hour after the start of dialysis, and after dialysis). If the body temperature is $\geq 37.3^{\circ} \mathrm{C}$ during dialysis and accompanied by symptoms such as cough, stop dialysis for epidemiological investigation. $\mathrm{n}$ the waiting area, use pictures and texts to make a one-meter interval sign, and paste cute little hedgehogs at the dividing points. The patient will feel love when seeing the little hedgehog, smile at each other, and tell jokes like don't be stabbed by the little hedgehog. The humorous signs continuously remind patients not to gather or get together, and stick to the onemeter interval regulation during diagnosis and treatment. Earnestly implement a series of prevention and control measures, ensure the safety of diagnosis and treatment in details, leave no dead ends in the measures, and leave no hidden dangers in the investigation, promote continuous improvement of work, be strict on guard and ensure the safety of dialysis patients.

(4) Health education The outbreak of the novel coronavirus epidemic was sudden, fierce, and covering a wide range. It caused serious damage and had great impact on people, especially the vulnerable group of maintenance hemodialysis patients, who suffered even more in this epidemic. The staff in the department have further strengthened health education, strengthened publicity and guidance during the epidemic, and collected, edited, and pushed the knowledge of epidemic prevention and control as soon as possible, and guided patients to treat the epidemic correctly and rationally, without being nervous, afraid, not believing or spreading rumors, be confident, actively cooperate with epidemic prevention and control work, and actively create an atmosphere of positive energy.

(5) Nursing quality contro/ During the novel coronavirus epidemic, the responsibilities of nursing staff have been highlighted. During the implementation of refined nursing interventions, nursing staff must strictly control the quality of nursing, and each nursing link should be intertwined to ensure the entire refined nursing process. At the same time, the nursing operation feedback information should be sorted out in the nursing process, and problems should be found and solved in time, so as to continuously improve the quality of nursing.

\subsubsection{Nursing Intervention During Dialysis}

(1) Strengthen education on epidemic prevention and contro/Educate patients about COVID-19 prevention knowledge, and correctly obtain information about the epidemic and related prevention and control and popular science knowledge, increase patients' awareness of COVID-19, and reduce their fear and strangeness, stabilize their psychological symptoms, and establish the confidence of patients to overcome the epidemic.

(2) Psychological intervention $₫$ Mental health management Since the outbreak of COVID-19, some dialysis patients have been under great psychological pressure and have different degrees of negative emotions, such as worry, anxiety, depression, etc. ${ }^{[7]}$. The pressure comes from the lack of knowledge about COVID-19, the worry that dialysis treatment will be interrupted due to the epidemic, the worry that they themselves or

Page $3 / 9$ 
their caregivers need to be isolated and feel helpless. Feeling dull and depressed, wearing a mask throughout the dialysis process, they worry about hypoglycemia, thirst and other discomforts. In order to effectively relieve the psychological pressure of dialysis patients and ensure the normal progress of hemodialysis treatment, nursing staff should pay attention to the patients' mental state, spirit, and emotion, actively communicate with patients, and adopt coping strategies. Inform patients that they can bring some candies, straws, etc. for emergencies. Use professional psychological care channels for dialysis patients, establish a platform for talk and exchange, face-to-face, WeChat groups, and video guidance to relieve stress, shoot anti-epidemic videos in dialysis rooms, improve immunity through exercise, and enhance anti-epidemic friendship through interaction. The video content and forms are diverse, such as playing harmonica, poetry recitation, dancing with fans during dialysis, leg raising exercise during dialysis, waving to cheer for Wuhan, talent show and competition, patients encouraging each other and enhancing friendship. Appropriate exercise can reduce dialysis patients' tension and anxiety, eliminate their life pressure and anxiety, make them feel more cared for, and at the same time enhance the nurse-patient relationship, improve the patients' happiness index, and then improve their self-confidence, and face life positively, optimistically, and strongly, achieve the goal of happy dialysis, and ultimately improve patients' compliance with dialysis treatment, and improve the quality of life of hemodialysis. $\nabla$ Emotional support Maintenance hemodialysis patients usually receive treatment three times a week, 4 hours each time. Most of the people they meet are medical staff and their surrounding wardmates. Nursing staff should adopt reasonable psychological intervention methods, actively care for patients and listen to patients' complaints, encourage patients to talk about their worries, ideas, treatment experience, and encourage patients to share self-dialysis experience. All these measures can effectively relieve the psychological pressure of patients Emotional fragility and depression of patients are one of the most common psychological problems of dialysis patients ${ }^{\circledR 8}$. Family members and dialysis partners are the main supporters of dialysis

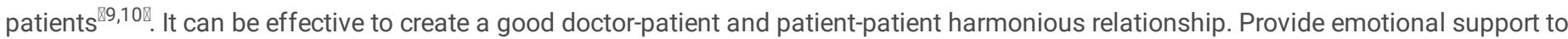
enhance the patients' ability to respond to COVID-19.

(3) Nursing operation norms Formulate corresponding nursing operation procedures and norms during the epidemic period, nurses must strictly follow the rules and procedures in their daily work, ensure that every nursing link meets the standard requirements, and strengthen monitoring of key nursing links. Due to the different degrees of psychological problems of dialysis patients, during the treatment process, patients will have negative psychological effects due to excessive stress, which will further affect their normal dialysis treatment. In order to effectively alleviate the psychological pressure of patients, nurses should "start from the heart". In the face of patients asking for help in pain and helplessness, nurses are no longer indifferent and cold, but enthusiastically help and patiently comfort every patient, with smile, love, enthusiasm, patience, sincerity, and responsibility. Nurses who are experienced and approved by patients provide treatment and care for patients, gain their trust and cooperation, create a good and harmonious nurse-patient relationship, and improve patient satisfaction, and thus win the honor for the department. After dialysis, the nursing staff assist or accompany patients with limited mobility to leave the dialysis room until they are handed over to the patients' family members to prevent adverse events.

\subsubsection{Nursing Intervention After Dialysis}

(1) Implement effective surface and air ventilation disinfection The dialysis room implements closed management, staggered on and off the machine, absolutely clears the field for dialysis. Between the two shifts, use $1000 \mathrm{mg} / \mathrm{L}$ chlorine disinfectant to wipe and disinfect the surface and ground, wipe and disinfect the high-frequency surface with $500 \mathrm{mg} / \mathrm{L}$ chlorine disinfectant ${ }^{[11]}$, strengthen detailed control, strengthen highfrequency surface disinfection management of various surfaces, and leave no dead ends. Ensure the air quality of the hemodialysis center, fully ventilate the departments, strengthen the ventilation between the two shifts of dialysis, and open the purification system throughout the whole process $^{[12]}$, perform terminal disinfection and keep records, and strictly control the closed-loop disinfection.

(2) Strengthen prevention and control, prevent the spread of the epidemic, emphasize home self-management Improve prevention and control awareness, strengthen prevention and control during the epidemic. Dialysis patients go to the hospital for dialysis 3 times a week, 4 hours each time, other times are discharge time, so home self-management is particularly important. Fully implement the patient management system, as for health education, comprehensively analyze, evaluate, plan, implement, and follow-up to complete stratified, individualized and systematic health guidance ${ }^{[13]}$, issue education brochures, strengthen the education of patients about prevention and treatment of COVID-19, actively

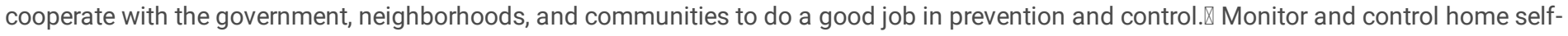
management, inform patients to pay attention to personal hygiene, keep the room clean and tidy, open windows for ventilation, keep indoor air circulation, and instruct them to keep warm during ventilation in winter to avoid catching colds. Try to go out as little as possible, avoid contact with people with coughs and fevers, and avoid group activities such as parties to prevent the occurrence of cluster epidemics. If you must go out, you must wear a mask correctly and wash your hands before wearing a mask to maintain hand hygiene. Distinguish the front and back of the mask, keep the dark side facing out, the metal strip on top. The mask should cover the mouth, nose, and chin, and the metal strip should be close to the bridge of the nose, so that the mask fits closely with the face. The key is to cover the mouth and nose completely. Don't pull the mask to the jaw, neck, etc. for the convenience of speaking and eating, and don't hang the mask on the arm, which will contaminate the inner layer of the mask and lose its protective effect. The mask should be replaced in time after it gets wet or contaminated. When removing the mask, be sure not to touch the outside of the mask with your hands. Hold the elastic cords on both sides of the mask with your hands, remove the mask from both ears, and fold the outside of the mask in and the inside out, and throw it into the designated trash can. Wash your hands frequently at home, do 
not spit anywhere, pay attention to cough etiquette, cover your mouth and nose with a tissue or elbow sleeve when coughing or sneezing, and after coughing or sneezing, going home or receiving express delivery, be sure to use soap and running water or alcohol based hand sanitizer to wash your hands correctly and develop good living and hygiene habits. $\bigotimes$ The patient's body temperature and respiratory symptoms of novel coronavirus infection are monitored and reported in real time. 区During the epidemic prevention and control period, dialysis patients should avoid changing the location of dialysis: if they need to change, it is recommended that they check with the original dialysis center after the epidemic is controlled and then return. If the patient returns from other provinces, it is necessary to know whether there is contact history with confirmed or suspected infection cases, fever cases and their families, and there is no isolation at home. This should be handled in accordance with relevant isolation requirements, and appropriate protective measures should be taken. $\nabla$ Keep detailed records of fixed and hospitalized dialysis patients and their families' home addresses, travel tracking and registration, and detailed records of the contact history of confirmed or suspected infected persons, home isolation or fever patients, etc. The above related information shall not be concealed.

(3) Diet instruction Nutrients are the basic material for human growth and development. Good nutrition not only provides the human body with immunity against diseases including COVID-19, but also is the primary guarantee for promoting disease recovery. Therefore, dialysis patients should consume enough calories (30凶35 kcal/ kg/ d) and high-quality protein $(1.0 \otimes 1.2 \mathrm{~g} / \mathrm{kg} / \mathrm{d}$ at least $50 \%$ comes from high-quality animal protein), and vegetarian food is not recommended. Properly control water intake, especially in the hot summer season, more attention should be paid to water intake. In order to reduce thirst, patients should refrain from too salty diet and alcohol, avoid drinking strong tea and strong coffee. If you are really thirsty, you can rinse your mouth with water and spit it up. Remember that the weight gain between two dialysis sessions should not exceed 3-5\% of your dry weight. Weight and blood pressure should be measured at fixed points every day. Accurately record and compare to assess whether the body's moisture is balanced, adhere to a low-salt, low-potassium and low-phosphorus diet, try not to eat raw food, separate cutting boards and tableware from raw and cooked food. Food should be washed and cooked. Wash hands before meals. During the epidemic, it is necessary to strengthen dietary hygiene and avoid eating overnight or unclean food, which may cause gastroenteritis and even peritonitis. Do not touch, buy and eat wild animals. Pay attention to the separation of raw and cooked food in the kitchen. Animal food should be cooked thoroughly. Family meals should be served individually or use public spoons and chopsticks to avoid mutual infection among family members.

(4) Proper body-building exercise to improve immunity Exercise has the effect of reducing negative emotions, enhancing physical fitness, and improving immunity. Exercise therapy uses the concept of sports medicine, based on the patient's functional situation. Active and passive exercises help patients improve their overall function and local function ${ }^{[14,15]}$. Refined nursing intervention combined with exercise therapy measures for hemodialysis patients can enhance the physical fitness of the patients through scientific and appropriate exercises. In turn, it helps the patient to develop a regular life routine, maintain the body's activity, produce effective stimulation to the nervous system, and make the patient's cerebral cortex in a relaxed state, thereby improving the patient's sleep quality. Effective stimulation makes the patient's cerebral cortex in a relaxed state, thereby improving the patient's sleep quality ${ }^{[16,17]}$, and also helping the patient to regulate biological rhythms and improve daytime sleepiness, and effectively promote the rationalization of body sleep and rest ${ }^{[18]}$. Thus reduce the adverse effects of hemodialysis and diseases, and improve the life function and quality of life of patients. Exercise therapy for hemodialysis patients can help patients form good exercise habits. Exercise can improve patients' nerve excitability, increase body happiness, enhance confidence in disease treatment, relieve physical and mental stress, and relieve negative emotions such as depression and anxiety ${ }^{[19]}$. Nursing staff use their professional knowledge to help patients understand the benefits of exercise based on the patient's own condition and physical fitness, assist patients in formulating scientific and reasonable exercise plans and personalized exercise programs, and gradually and slowly increase exercise time and intensity through a step-by-step method. With scientific nutrient supply, it helps patients maintain their energetic state, strengthen their physical functions, restore social functions, better integrate into society, increase participation in social activities, and improve patients' physical and mental health and quality of life ${ }^{[20]}$.

(5) Improve spiritual realm and enrich cultural life During the epidemic, maintenance hemodialysis patients must love life more, care about current affairs, keep their mind open and at ease, overcome anxiety and fear, arrange life in a reasonable and orderly manner, and cultivate more hobbies, such as painting, calligraphy, embroidery, music, etc., let interests and hobbies arouse self-positive motivation, watch (listen to) more comedies, positive programs (books), watch (listen to) less or not (listen to) tragedy or negative programs (books) to enrich spiritual and cultural life.

(6) Popularize information technology and keep pace with the times In recent years, with the improvement of the medical security system and medical conditions, the average life expectancy of human beings has increased, and the number of elderly patients with chronic renal failure has continued to increase. Therefore, the number of elderly patients undergoing maintenance hemodialysis is also increasing [21]. People are gradually realizing that improving the quality of care for elderly patients with uremic maintenance hemodialysis is an important guarantee for improving the quality of life of elderly patients with uremic ${ }^{\text {[22] }}$. With the popularization of information technology, mobile phones have become an indispensable configuration in people's daily lives, and people's lifestyles have also changed. However, elderly maintenance hemodialysis patients lack the mastery of new technologies and skills. Most of the patients' mobile phones are phones specially designed for the elderly, and some patients even do not have a mobile phone, and they don't know anything about the Internet and WeChat. In order to enhance patients' ability to cope with epidemics and emergencies, nursing staff strengthen communication with patients, carefully, patiently and meticulously

Page $5 / 9$ 
guide them to learn to use online platforms, WeChat, QQ, online appointments, etc., and patients actively learn new technologies and master new skills. Some patients even bought a new smartphone for this purpose and actively cooperated with various measures, in order to overcome obstacles and respond effectively to epidemics or emergencies.

\subsubsection{Statistical Methods}

172 cases of maintenance hemodialysis patients in our hospital were selected. From February 1, 2020 (before intervention) to July 1, 2020 (after intervention), SCL-90 questionnaire survey method was used for retrospective analysis. Use the "questionnaire star" to scan the QR code with WeChat to fill in the questionnaire, which are distributed, explained and collected by a designated person. There were 172 questionnaires in total, 172 were returned, and the recovery rate was $100 \%$.

Statistical analysis uses SPSS21 software, and measurement data analysis uses frequency, mean, standard deviation, and two-sample mean t test.

\section{Results}

\subsection{General information}

172 cases of maintenance hemodialysis patients in our hospital were selected for questionnaire survey from February 1, 2020 to July 1, 2020, including 96 males and 76 females, aged 26 - 84 years, with an average age of $(53.13 \pm 12.79)$ years; dialysis age was more than 2 months; disease types: 46 cases of chronic glomerulonephritis, 56 cases of high blood pressure kidney cases, 65 cases of diabetic nephropathy, 5 cases of lupus nephritis; education level: 22 cases of junior high school and below, 123 cases of senior high school and technical secondary school, and 27 cases of junior college and above.

\subsection{Comparison of SCL-90 scores of maintenance hemodialysis patients before and after refined nursing intervention}

Before the psychological intervention of hemodialysis patients, the score of depression in the 10 factors of SCL-90 score was ( $2.38 \pm 0.30)$, and the score of anxiety was $(2.29 \pm 0.27)$; the depression after intervention was $(1.59 \pm 0.35)$, and the score of anxiety was $(1.57) \pm 0.23)$. ( $t$ depression $=6.02, p<0.01) t$ anxiety $=7.99, p<0.01)$. The difference in depression and anxiety scores was statistically significant. After the intervention, the patient's depression and anxiety alleviated. See Table 1. Both nucleic acid test results of all patients were negative.

Table 1

Comparison of SCL-90 scores $(\mathrm{x} \pm \mathrm{s})$

\begin{tabular}{|c|c|c|c|c|c|c|c|c|c|c|}
\hline Group & Somatization & $\begin{array}{l}\text { Obsessive } \\
\text { compulsive } \\
\text { disorder }\end{array}$ & $\begin{array}{l}\text { Interpersonal } \\
\text { sensitivity }\end{array}$ & depressed & anxious & hostile & terror & Paranoia & Psychotic & $\begin{array}{l}\text { Additional } \\
\text { factors }\end{array}$ \\
\hline $\begin{array}{l}\text { The } \\
\text { outbreak } \\
\text { began }\end{array}$ & $1.44 \pm 0.17$ & $1.59 \pm 0.31$ & $2.52 \pm 0.22$ & $\begin{array}{l}2.38 \pm \\
0.30\end{array}$ & $\begin{array}{l}2.29 \pm \\
0.27\end{array}$ & $\begin{array}{l}2.20 \pm \\
0.50\end{array}$ & $\begin{array}{l}2.29 \\
\pm \\
0.43\end{array}$ & $\begin{array}{l}1.51 \pm \\
0.28\end{array}$ & $\begin{array}{l}1.83 \pm \\
0.44\end{array}$ & $\begin{array}{l}1.52 \pm \\
0.58\end{array}$ \\
\hline $\begin{array}{l}\text { epidemic } \\
\text { situation }\end{array}$ & $1.42 \pm 0.15$ & $1.48 \pm 0.30$ & $1.65 \pm 0.29$ & $\begin{array}{l}1.59 \pm \\
0.35\end{array}$ & $\begin{array}{l}1.57 \pm \\
0.23\end{array}$ & $\begin{array}{l}1.59 \pm \\
0.33\end{array}$ & $\begin{array}{l}1.43 \\
\pm \\
0.29\end{array}$ & $\begin{array}{l}1.50 \pm \\
0.25\end{array}$ & $\begin{array}{l}1.80 \pm \\
0.40\end{array}$ & $\begin{array}{l}1.50 \pm \\
0.61\end{array}$ \\
\hline T Value & 0.97 & 0.48 & 5.14 & 6.02 & 7.99 & 4.58 & 6.96 & 1.02 & 0.97 & 0.56 \\
\hline P Value & $\mathrm{P} \otimes 0.05$ & $P \otimes 0.05$ & $P \otimes 0.01$ & $P \otimes 0.01$ & $P \otimes 0.01$ & $\begin{array}{l}\mathrm{P} \\
\bigotimes 0.01\end{array}$ & $\begin{array}{l}P \\
\bigotimes 0.01\end{array}$ & $P \otimes 0.05$ & $P \otimes 0.05$ & $\mathrm{P} \otimes 0.05$ \\
\hline
\end{tabular}

\section{Discussion}

According to the survey results, after nursing intervention in the July epidemic stage, the patients' anxiety and depression levels were significantly improved, with statistical differences. Both nucleic acid test results were negative, indicating that the patient had no COVID-19 infection. Compared with other types of diseases, "COVID-19" is more special and dangerous ${ }^{[23]}$, and it poses a greater threat to the health of the patient and the surrounding population ${ }^{[24]}$. In addition to adopting scientific and effective treatment plan, it should be supplemented with corresponding nursing intervention. Apply the refined nursing intervention model to the clinical treatment of maintenance hemodialysis patients, based on improving the nursing concepts of nursing staff, make nursing staff fully aware of the importance and value of the application of refined nursing model, further strengthen nursing staff's operating norms, improve nursing quality ${ }^{[25]}$, and fundamentally enrich nursing staff's theoretical knowledge and improve clinical practice capabilities to achieve refined nursing intervention for patients with maintenance hemodialysis and improve the overall quality of care. Strengthen communication with patients and their families, so that they fully understand 
the COVID-19 related knowledge and precautions, control the spread of infectious diseases, and ensure the safety of patients and surrounding people ${ }^{[26]}$.

Long-term maintenance hemodialysis treatment causes great economic and mental stress to patients, which can lead to negative emotions and reduced quality of life in patients ${ }^{[27,28]}$. As one of the new models of modern nursing, the core of the refined nursing intervention model is humanistic care, which aims to provide patients with the highest quality nursing services, effectively improve the quality of life of patients, and maximize their benefits ${ }^{[29]}$. This shows that maintenance hemodialysis patients receiving refined nursing intervention model can help promote their health behavior compliance, correct their previous cognitive misunderstandings, improve their self-management ability and information practice awareness, improve their quality of life, and reduce negative emotions, reduce the incidence of complications, and help maintain a good nurse-patient relationship, paving the way for the full implementation of nursing work ${ }^{[30]}$. In addition, the full companionship of the responsible nurse in the entire nursing work can promote the patient's psychological satisfaction of being cared for and reduce the degree of unhealthy emotions such as tension. The emotional communication and careful care during the dialysis process can gain the trust of the patient, reduce pain, improve their psychological and physical comfort ${ }^{[31]}$. Adjust their dietary structure according to the characteristics and progress of the patient's condition, limit the intake of salt, potassium, phosphorus, and water, which help to accelerate the prognosis of the disease and relieve the clinical symptoms ${ }^{[32,33]}$. Therefore, through the above measures, the psychological and physical comfort of patients can be improved, and the two influence each other and cause each other to jointly improve the quality of life of patients, strengthen their belief in rehabilitation, and benefit the prognosis ${ }^{[34]}$.

\section{Conclusion}

In summary, giving maintenance hemodialysis patients meticulous care intervention during the novel coronavirus epidemic can improve their health behavior compliance, regulate negative emotions, improve quality of life, reduce complications, and fundamentally control the occurrence of incidents resulted from poor care. It helps to enhance the nurse-patient relationship and improve patient satisfaction, which has high clinical promotion value.

\section{Abbreviations}

SCL-90: Symptom Checklist90

COVID-19: Coronavirus Disease 2019

\section{Declarations}

\section{Ethics approval and consent to participate:}

This study was conducted in accordance with the Declaration of Helsinki and approved by the ethics committee of Chaoyang Hospital Affiliated to Capital Medical University.

\section{Consent to publication:}

All authors final approval of the version to be published.

\section{Availability of data and materials:}

The datesets used or analyzed during the current study are available from the corresponding author on reasonable request.

\section{Competing interests:}

All of the authors had no any personal, financial, commercial, or academic conflicts of interest separately.

\section{Funding:}

None.

\section{Authors Contribution}

Conception and design of the research: Zhang QL, Wang S. Acquisition of data: Zhang Y. Analysis and interpretation of the data: Meng F. Statistical analysis : Zhang Y, Meng F. Obtaining financing: None. Writing of the manuscript: Zhang QL, Wang S. Critical revision of the manuscript for intellectual content : Zhang QL, Wang S. 
N/A.

\section{References}

[1] Expert group on novel coronavirus novel coronavirus pneumonia prevention and control in China Preventive Medicine Association. Latest understanding of epidemiology of new coronavirus pneumonia[J/OL].Chinese Journal of viral diseases,2020,10®3凶ख81-87.

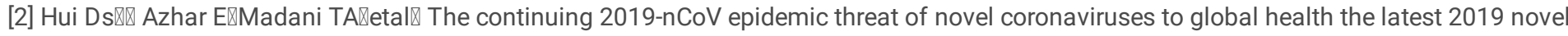
coronavirus outbreak in Wuhan,China[J].Int J Infect Dis,2020,91:264-266.

[3]Lipsitch M,Swerdiow DL,Finelli L.Defining the epide miology of covid 19 studies needed[J/OL].N Engl J Med,2020.DOL.org/10.1056/NFJMp2002125.[Epub ahead of print]

[4]Zhou J, Yan JJ, Tong H.Management and practice of novel coronavirus pneumonia during psychological control and prevention during hemodialysis patients[J]Integrated traditional Chinese and Western medicine nursing,(Chinese and English),2020,6冈3冈ख139-141.

[5]Qiu XY, Li AF.Clinical study on psychological nursing intervention of maintenance hemodialysis patients[J]Electronic Journal of practical clinical nursing,2020,5囚3囚:86.

[6]Tang QP, Cheng ZH. Yuan AH,et al.Application and analysis of SCL-90 in China

[J]Chinese Journal of Clinical Psychology, 1999ه1囚:16-20.

[8]Cui HX, Ding HJ, Zhang YM, et al.Novel coronavirus pneumonia in different periods of dialysis patients psychological state and nursing countermeasures[J] Qilu Journal of nursing $₫ 2020 \rrbracket 26 \rrbracket 12 \otimes \nabla 61-63$.

[9]Tao CH, Li Y.Study on quality of life, negative emotion and coping style of maintenance hemodialysis patients[J] Journal of clinical kidney disease $2020 \otimes 20 \otimes 2 \otimes \Downarrow 121-124$.

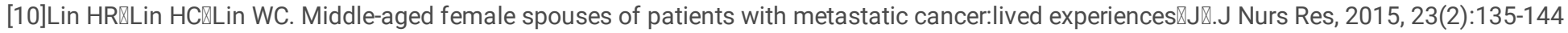

[11]You CX, Zhang CP, Zhang MJ.Qualitative study on the care compliance experience of main caregivers of patients with advanced cancer in

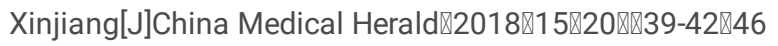

[12]Wen FC, Cai Mo L.Strengthen environmental disinfection management of blood purification room to prevent and control hospital infection[J]Digest World Latest Med Inf (continuous e-journal),2019,19(40): 1-2,18.

[13]Yao X, Zhang BL, Gong YX, et al.】Hospital air purification management specification WS / t368-2012 IImplementation survey[J]Chinese Journal of infection control,2019,18(11):1032-1037.

[14]Qin Y, Tian XHm Liu CP, et al.The effect of maintenance hemodialysis on the quality of life of hemodialysis patients[J]Beijing medicine $\mathbb{Z}$ 2019®41ه7هष618-621.

[15]Jiang L, Wei WJ,Li P.Study on the application effect of comfortable nursing in hemodialysis nursing[J]Chinese Medical Guide,2016,18(9):963-964.

[16]Liang R, Cao WJ.Research progress of hypotension and exercise therapy in hemodialysis patients[J]Nursing research,2018,32(24):20-23.

[17]Li YY, Zhu HD, Zhang L, et al.Effect of exercise team on improving quality of life, sleep quality and dialysis adequacy in hemodialysis patients[J]Journal of Xinjiang Medical University,2018,41(10):41-44,48.

[18]Chen YQ, Luo L.Effect of holistic nursing intervention on quality of life and clinical efficacy of hemodialysis patients with chronic renal failure[J]Hebei Medicine2016,22(8):1370-1372.

[19]Jiachun LU,Bai X,Lei X,et al. Acupuncture Combined with Kinesitherapy in Treating 40 Cases of Senile Stroke Patients with Sleep Disorder[J]. Rehabilitation Medicine,2016,26(2):17.

[20]YS, Wan F.Research progress of exercise patterns in maintenance hemodialysis patients[J]Blood purification in China,2016,15(5):312-314. 
[21]Kuznik BI,Davydov SO,Stepanov AV,et al. The Effect of Kinesitherapy Exercises on the Level of Irisin among Females with Cardiovascular diseases depending on the body mass and hormonal status.[J]. Patologicheskaia Fiziologiia I Eksperimentalnaia Terapiia,2016,60(4):47-51.

[22]Guo QZ.Puncture and nursing of artificial vascular fistula in elderly hemodialysis patients[J]Chinese Journal of Geriatrics, 2016,18 (6) :370.

[23]Wu LX, Gao L, Fang KN.Clinical nursing of maintenance hemodialysis in elderly patients with uremia[J]Baotou medicine, $2014,(1)$ :46-48.

[24] He CM, Meng LX, Chen JF,et al.Study on the effectiveness of nursing emergency management in the treatment of sudden infectious diseases[J]Jilin medicine, 2017, 38 (6) :1191-1193.

[25]Ma MM, Xiao AQ, You MY, et al.Effect of family centered health education on the isolation compliance of parents of children with respiratory infectious diseases

[J]Chinese Journal of modern nursing,, 2016, 22 (10):1379-1382.

[26]Zhang LL, Ren Z, Bao ZY, et al.Investigation on the needs of nursing interns in infectious diseases hospital for professional quality of Teachers[J]Chinese Journal of Modern Nursing, 2014, 20 (4) :459-461.

[27]Li JL, Zeng GY, Ling Y, et al.Ethical conflicts and Countermeasures in the practice of compulsory medical care for patients with sudden infectious diseases

[J]Records of snake 2014, 26 (1) :137-139.

[28] Yang MF, Zhu H, Guo XJ, et al.Analysis of the influence of knowledge, attitude and practice health education mode on self diet management quality of maintenance hemodialysis patients[J] Health management in China, 2018, 35 (5) :391-393.

[29] GAMBOA J L, PRETORIUS M, SPRINKEL K C, et al.Angiotensin converting enzyme inhibition increases ADMA concentration in patients on maintenance hemodialysis a randomized cross-over study[J].Bmc Nephrology, 2015, 16 (1):167.

[30] Zhao $\mathrm{CH}$, Li P, Kong QH, et al.Effect of detail optimization nursing on artificial vascular fistula and self-efficacy of hemodialysis patients

[J]Blood purification in China, 2015, 14 (8) :499-502.

[31] Feng XF, Jiang Q, Zeng Y, et al.Application of quality control circle in continuous improvement of hemoglobin compliance rate in hemodialysis patients

[J]Blood purification in China, 2018, 17 (4) :265-267.

[32] HSU C W, YEN T H, CHEN K H, et al.Effect of blood cadmium level on mortality in patients undergoing maintenance hemodialysis[J].Medicine, 2015, 94 (42) :e1755.

[33] Xu L, Xiao J.Effect of continuous nursing on self-management behavior and quality of life of maintenance hemodialysis patients[J]Chinese medical equipment, 2018, 15 (5) :128-130.

[34] Zhang YJ, Xue GF, Yang YJ, et al.Influence of empowerment education on health literacy and self-management ability of maintenance hemodialysis patients

[J]Blood purification in China, 2018, 17 (3) :193-196.

[35] Xie Ke, Wang Min. Research on the effect of refined nursing model on elderly patients with diabetic nephropathy on hemodialysis[J]. Modern Journal of Integrated Traditional Chinese and Western Medicine, 2017, 26 (4): 446-448.

\section{Supplementary Files}

This is a list of supplementary files associated with this preprint. Click to download.

- Questionnaire.docx

- Questionnaire.docx 